\title{
Synchronous Sensing Analysis Based on Nonmodified Multi- Metal Sensor for Five Tastes
}

\author{
Lin Lu*, Xianqiao Hu, Zhiwei Zhu* \\ Rice Product Quality Supervision and Inspection Center, Ministry of Agriculture, China National Rice \\ Research Institute, Hangzhou 310006, China \\ *E-mail: luzi0522@163.com
}

doi: $10.20964 / 2018.06 .41$

Received: 27 January 2018 / Accepted: 23 March 2018 / Published: 10 May 2018

\begin{abstract}
This paper deals with a synchronous sensing analysis for five tastes namely sweetness, bitterness, sourness, saltiness, astringency by qualitatively and quantitatively analyzing taste stimuli with nonmodified multi-metal sensor, which has the advantage of being rapid, easy and simultaneous. Eight metal electrodes that were easily available constituted the electrode array. Cyclic voltammetry (CV) and differential pulse voltammetry (DPV) were employed. The original voltammetric signals were transformed by Continuous Wavelet Transform (CWT) in order to reveal more feature information for sensing taste stimuli. The data of feature points from the transformed signal as the input were used for neural network model. Polar Phasor Approach was used to classify and locate single taste. It was resulted that Layer-Recurrent neural network could effectively identify the types of mixed tastes. The response areas of eight electrodes for five tastes in oxidation part and reduction part of CV and DPV were visually characterized. Combining CV with DPV, the accuracies of the training set and test set were both more than that of single voltammetry, confirming that Back Propagation neural network could quantitatively analyze each taste of the mixture. Moreover, the advantages of nonmodified sensor compared with modified sensor were discussed. It was concluded that synchronous sensing analysis has strong ability to sensing the five tastes accurately and simultaneously as human gustation.
\end{abstract}

Keywords: Taste stimulus; Synchronous sensing; Voltammetry; Nonmodified sensor

\section{$\underline{\text { FULL TEXT }}$}

(C) 2018 The Authors. Published by ESG (www.electrochemsci.org). This article is an open access article distributed under the terms and conditions of the Creative Commons Attribution license (http://creativecommons.org/licenses/by/4.0/). 